\title{
ADRENOCORTICAL CONTROL OF SODIUM AND POTASSIUM EXCRETION IN THE NEWBORN PERIOD ${ }^{1}$
}

\author{
BY ROBERT KLEIN \\ (From the Department of Pediatrics, the Johns Hopkins University School of Medicine, and \\ the Harriet Lane Home of the Johns Hopkins Hospital, Baltimore, Md.)
}

(Submitted for publication August 14, 1950; accepted, January 2, 1951)

The role of the adrenals in response to disease during the neonatal period has long been a subject of speculation. The anatomical peculiarities of the adrenal cortex in this period are well known. Jaudon (1) has suggested that transient deficiencies in adrenocortical function occur in the newborn period. However, few experimental studies have been reported. Venning (2) has reported that the newborn under one week of age excretes less neutral reducing lipids in response to the administration of adrenocorticotropic hormone (hereinafter called ACTH) than does the older infant. We have found that during the first week of life the decrease in circulating eosinophils after the administration of ACTH is less than in older infants (3).

The author has suggested that stimulation of the newborn adrenal does not cause sodium retention (4). In the present study ACTH was given to newborn infants to study the effect of the adrenal cortex on electrolyte control.

\section{METHODS}

Eleven infants in the premature nursery of the Harriet Lane Home and three full term infants three weeks of age or under and one six months old full term infant on the general ward of the Harriet Lane Home were studied during the winter of 1949-50. The premature infants were all healthy with the exception of two who were being treated for retrolental fibroplasia. ${ }^{2}$ The six months old infant was being observed in the hospital for congenital nonhemolytic jaundice. Two of the other three patients on the infants ward had recovered from the acute pneumonitis for which they were admitted. The third patient had congenital glaucoma.

1 This work was made possible by a grant from the American Cancer Society for studies on the relationship of the pituitary hormones, thyroid hormone, and the steroid hormones of the adrenal glands and gonads to normal and abnormal growth, and by a grant from the Commonwealth Fund for the study of endocrine problems in childhood.

2 These were treated under the supervision of Drs. W. and E. Owens.
Complete urine collections were made with the few exceptions to be noted in the tables. Soft rubber tubing fitting snugly over the penis was used for collecting urine from the males. Urine was collected from female patients by placing the infant in an ordinary bassinet with the head of the bassinet slightly elevated, and with the patient's buttocks over a Buchner funnel. The patients were supported in this position by slings supporting their lower thighs. Five cc. of $25 \%$ acetic acid were added to each day's urine collection.

Patients were weighed routinely three times a week on the regular balances of the wards and nursery. Weights are recorded for the beginning of the daily periods.

Eosinophil counts were made by the method of Randolph (5). Sodium and potassium in the urine were measured by an internally compensated Perkin-Elmer flame photometer. 17-Ketosteroids and neutral reducing lipids were measured by methods previously described (6). Nitrogen in the urine was determined by nesslerization. Serum and stool electrolytes were not determined. Dietary intake was calculated from conventional tables and random samples were analyzed for sodium and potassium content using the wet ashing method of Wallace (7) and measuring with a flame photometer. The volume of feeding mixture in each bottle was accurately measured, but when any of the bottle was refused, the amount was estimated by the nurse using the calibrations on the bottle. Fortunately, this occurred rarely.

$\mathrm{ACTH}^{3}$ was given to 11 patients for one day only, one receiving $10 \mathrm{mg}$. intramuscularly every six hours, one $10 \mathrm{mg}$. every eight hours, six $5 \mathrm{mg}$. every six hours, two $2 \mathrm{mg}$. every six hours, and one $1 \mathrm{mg}$. every six hours. Two other patients received ACTH four times daily for periods of seven and 10 days respectively.

When desoxycorticosterone acetate (hereinafter called DCA) or cortisone were used they were given as a single intramuscular injection at the beginning of the daily period.

\section{RESULTS}

Seven of the newborn infants tested with ACTH for one day were prematures who had weighed between 1,210 and 1,800 grams at birth (Cases $1-4,10,11,14)$. At the time of the study their weights were between 1,330 and 3,125 grams.

\footnotetext{
${ }^{3}$ Kindly supplied by Dr. J. R. Mote of the Armour Laboratories, Chicago, Illinois.
} 
The oldest was 41 days old. In addition three full term infants three weeks of age or less and weighing between 3,150 and 4,150 grams were studied. Sodium retention on the day of ACTH administration in these cases was minimal or non-existent. However, in all but one of the patients there was a significant increase in excretion of sodium in the urine on the day following administration of ACTH. This loss in the urine was often greater than the calculated intake for the day. It was accompanied by an increase in urinary volume. There was usually a decreased rate of the weight gain in this period. The one infant who did not show this sodium loss was one of those receiving the smallest doses of ACTH.

The effect of ACTH on potassium excretion was more variable. In some infants (and these usually were among the larger who received the larger doses) there was an increased urinary excretion of potassium on the same day that ACTH was given whereas there was no change in urinary sodium until the following day. In other infants, potassium excretion did not change or else varied directly with the volume of urine. The initial rise in potassium excretion was invariably associated with increased urinary nitrogen excretion. Usually the amount of nitrogen excretion did not indicate that there was sufficient protein catabolism to account for the potassium lost. In several cases there was a secondary rise in potassium excretion two or three days after ACTH was given. This, too, was always associated with a secondary rise in nitrogen excretion.

The nitrogen excretion in the urine showed a uniform rise when ACTH was administered. This occurred either on the day ACTH was given or on the following day.

In all those infants over one week of age on whom counts were made, there was a fall in circulating eosinophils irrespective of the dose of ACTH. Since these results were in accord with previous work (3), such counts were made only in the first few studies. It is of importance to note that there was the expected rise in excretion of 17 -ketosteroids and neutral reducing lipids after administration of ACTH. On continued stimulation, the 17-ketosteroid levels rose at times to normal adult female levels and the neutral reducing lipids far exceeded normal adult levels.
Since in these newborns ACTH failed to cause sodium retention which usually occurs in older children and adults, it is important to note the effect of DCA in this group of patients. The two newborns who were given $5 \mathrm{mg}$. of DCA in a single intramuscular injection and the one who was given $2 \mathrm{mg}$. showed a definite decrease in urine volume and sodium excretion (Cases 9-11). The patient who received only $1 \mathrm{mg}$. of DCA showed no definite sodium retention although his urine volume did decrease. DCA caused no increase in potassium excretion, but instead, in some cases, there was a decrease parallel to the reduction in urine volume.

Two premature infants were given cortisone (Cases 13, 14). When $50 \mathrm{mg}$. were given to the first infant there was no significant change in electrolyte excretion. The second infant who received $25 \mathrm{mg}$. of cortisone showed marked urinary retentions of water, sodium and potassium. This

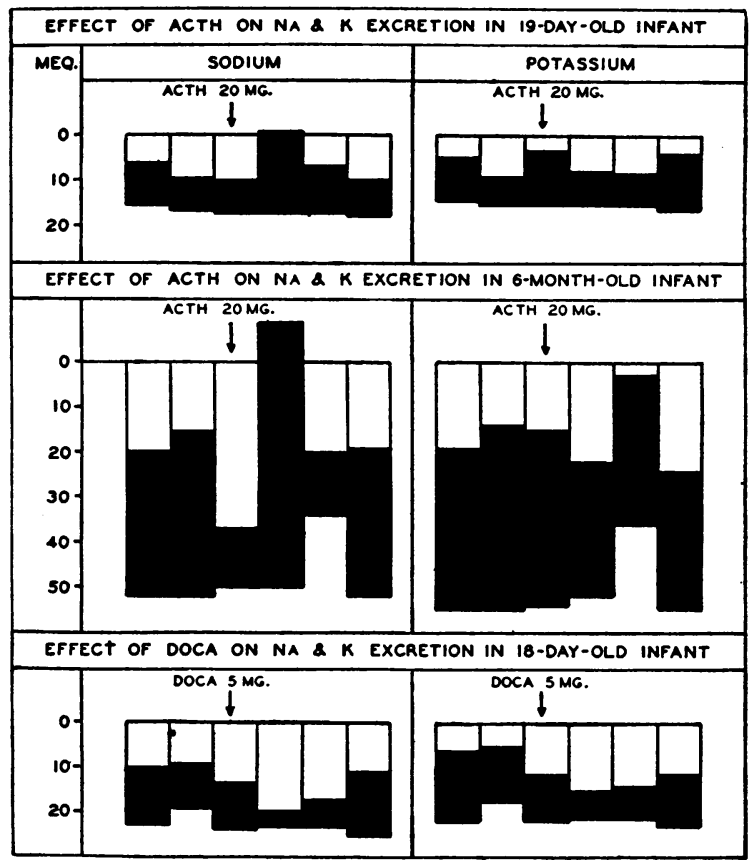

Fig. 1. Electrolyte Balances of a 19 Day Old Infant Given ACTH, and of a Six Month Old Infant Given ACTH and an 18 Day Old Infant Given DCA

Each column represents one day. Intakes are plotted down from the zero line, and urinary excretion (blacked in) is plotted up from the intake. The resultant white spaces below the zero line represent positive balances and the blacked in areas above the zero line represent negative balances. No allowance is made for stool or sweat electrolytes. 
infant then was given $\mathrm{ACTH}$ and responded to it in the same manner as the other newborns.

In addition to the ten infants who were treated with ACTH for only 24 hours, two premature infants were treated with ACTH for seven and 10 days respectively in an experimental attempt to influence retrolental fibroplasia (Cases 7,8). Neither of these patients showed any signs of escape from the effects of ACTH on electrolyte balance. The increased sodium excretion continued for two or three days after discontinuing ACTH. One of these prematures developed diarrhea while being treated with ACTH (Case 8). $\mathrm{He}$ continued to pour out more sodium in spite of the fact that there was probably an increased loss of sodium in the stools. His weight fell sharply and it was felt that the magnitude and rapidity of the loss were greater than would be expected from the relative mildness of his diarrhea. Three days after ACTH was discontinued conservation of sodium occurred due to a decrease in the urinary excretion. Until this time the additional amounts of parenteral saline received were excreted within 24 hours in the urine. He was able to retain potassium better even while getting $\mathrm{ACTH}$, although one day while he was having diarrhea the urinary excretion exceeded the intake. The urinary volume remained high until one week after

TABLE I

Electrolyte and steroid data for 15 infants studied

\begin{tabular}{|c|c|c|c|c|c|c|c|c|c|c|c|c|}
\hline \multirow{2}{*}{ Case } & \multirow{2}{*}{ Day } & \multirow{2}{*}{$\mathbf{R x}$} & \multirow{2}{*}{ Wt. } & \multicolumn{3}{|c|}{ Intake } & \multicolumn{4}{|c|}{ Urinary output } & \multicolumn{2}{|c|}{ Urinary steroids } \\
\hline & & & & Vol. & $\mathbf{N a}$ & $\mathbf{K}$ & Vol. & $\mathrm{Na}$ & $\mathbf{K}$ & $\mathbf{N}$ & $17 \mathrm{KS}$ & $\begin{array}{c}\text { Neutral red. } \\
\text { lipids }\end{array}$ \\
\hline $\begin{array}{l}\text { Case } 1 \\
18 \text { days } \\
\text { birth wt. } \\
1,765 \text { grams }\end{array}$ & $\begin{array}{l}1 \\
2 \\
3 \\
4 \\
5\end{array}$ & $\begin{array}{c}0 \\
0 \\
\text { ACTH } \\
4 \\
0 \\
0\end{array}$ & $\begin{array}{l}\text { grams } \\
1,600 \\
1,660 \\
1,775\end{array}$ & \begin{tabular}{r|}
$c c$. \\
82 \\
205 \\
210 \\
210 \\
210
\end{tabular} & \begin{tabular}{|c|} 
meq \\
5.5 \\
13.9 \\
14.3 \\
14.3 \\
14.3
\end{tabular} & \begin{tabular}{|c|} 
meq \\
4.6 \\
11.5 \\
11.7 \\
11.7 \\
11.7
\end{tabular} & $\begin{array}{l}c c . \\
84 \\
72 \\
70 \\
65 \\
54\end{array}$ & \begin{tabular}{|c|}
$m e q$ \\
4.5 \\
4.8 \\
4.8 \\
5.2 \\
3.1
\end{tabular} & $\begin{array}{c}\text { meq } \\
3.8 \\
4.2 \\
5.5 \\
3.5 \\
3.1\end{array}$ & \begin{tabular}{|c|} 
grams \\
0.29 \\
0.34 \\
0.38 \\
0.32 \\
0.17
\end{tabular} & $\begin{array}{l}\text { mg. } \\
0.54 \\
0.44 \\
0.94 \\
0.54 \\
0.32\end{array}$ & $\begin{array}{l}\text { mg. } \\
0.34 \\
0.31 \\
0.57 \\
0.56 \\
-\end{array}$ \\
\hline $\begin{array}{l}\text { Case } 1 \\
\text { at } 33 \text { days }\end{array}$ & $\begin{array}{l}1 \\
2 \\
3 \\
4 \\
5 \\
6\end{array}$ & $\begin{aligned} & 0 \\
& 0 \\
& \text { ACTH } \\
& 4 \\
& 0 \\
& 0 \\
& 0 \\
& 0\end{aligned}$ & $\begin{array}{l}2,400 \\
2,465 \\
2,600\end{array}$ & $\begin{array}{l}430 \\
510 \\
510 \\
450 \\
510 \\
485\end{array}$ & $\begin{array}{l}18.0 \\
18.0 \\
18.0 \\
18.0 \\
18.0 \\
18.0\end{array}$ & $\begin{array}{l}16.9 \\
16.9 \\
16.9 \\
16.9 \\
16.9 \\
16.9\end{array}$ & $\begin{array}{l}\text { incon } \\
240 \\
240 \\
203 \\
194 \\
238\end{array}$ & $\begin{array}{r}6.7 \\
8.6 \\
11.8 \\
7.4 \\
8.1\end{array}$ & $\begin{array}{r}\text { collec } \\
6.5 \\
8.9 \\
9.1 \\
7.4 \\
8.8\end{array}$ & $\begin{array}{l}\text { tion } \\
0.87 \\
1.45 \\
0.13 \\
0.08 \\
0.67\end{array}$ & $\begin{array}{l}0.85 \\
1.43 \\
1.22 \\
0.86 \\
0.97\end{array}$ & $\begin{array}{l}0.29 \\
0.28 \\
0.28 \\
0.26 \\
0.29\end{array}$ \\
\hline $\begin{array}{l}\text { Case } 2 \\
9 \text { days } \\
\text { birth wt. } \\
1,430 \text { grams }\end{array}$ & $\begin{array}{l}1 \\
2 \\
3 \\
4 \\
5 \\
6 \\
7 \\
8\end{array}$ & $\begin{aligned} 0 \\
0 \\
0 \\
0 \\
\text { ACTH } \\
8 \mathrm{mg} . \\
0 \\
0 \\
0\end{aligned}$ & $\begin{array}{l}1,330 \\
1,345 \\
1,430 \\
1,495\end{array}$ & $\begin{array}{l}126 \\
146 \\
150 \\
175 \\
180 \\
180 \\
205\end{array}$ & $\begin{array}{r}8.3 \\
9.6 \\
9.9 \\
11.5 \\
11.9 \\
11.9 \\
13.5\end{array}$ & $\begin{array}{r}7.0 \\
8.0 \\
8.3 \\
9.6 \\
9.9 \\
9.9 \\
11.5\end{array}$ & $\begin{array}{r}60 \\
46 \\
50 \\
60 \\
110 \\
84 \\
33\end{array}$ & \begin{tabular}{r|}
3.1 \\
3.2 \\
2.5 \\
3.3 \\
11.2 \\
7.2 \\
2.8
\end{tabular} & $\begin{array}{l}3.0 \\
4.3 \\
4.3 \\
5.4 \\
4.1 \\
5.6 \\
6.3\end{array}$ & \begin{tabular}{|l|}
0.27 \\
0.31 \\
0.26 \\
0.30 \\
0.41 \\
0.31 \\
0.38
\end{tabular} & $\begin{array}{l}0.01 \\
0.00 \\
0.04 \\
0.50 \\
0.39 \\
0.00 \\
0.37\end{array}$ & $\begin{array}{l}0.06 \\
0.06 \\
0.05 \\
0.12 \\
0.14 \\
0.11 \\
0.08\end{array}$ \\
\hline $\begin{array}{l}\text { Case } 2 \\
\text { at } 41 \text { days }\end{array}$ & $\begin{array}{l}1 \\
2 \\
3 \\
4 \\
5 \\
6 \\
7\end{array}$ & $\begin{aligned} & 0 \\
& 0 \\
& \text { ACTH } \mathbf{8} \\
& 0 \\
& \mathbf{0} \\
& \mathbf{0} \\
& \mathbf{0} \\
& \mathbf{0}\end{aligned}$ & $\begin{array}{l}2,125 \\
2,235 \\
2,340 \\
2,450\end{array}$ & $\begin{array}{l}375 \\
345 \\
370 \\
390 \\
360 \\
390 \\
390\end{array}$ & $\begin{array}{l}15.0 \\
13.8 \\
14.8 \\
15.6 \\
14.4 \\
15.6 \\
15.6\end{array}$ & $\begin{array}{l}13.9 \\
12.8 \\
13.7 \\
14.4 \\
13.4 \\
14.4 \\
14.4\end{array}$ & $\begin{array}{l}188 \\
152 \\
218 \\
200 \\
160 \\
150 \\
170\end{array}$ & \begin{tabular}{r|}
7.1 \\
6.4 \\
11.1 \\
13.6 \\
7.0 \\
6.6 \\
8.3
\end{tabular} & $\begin{array}{r}11.1 \\
9.9 \\
12.6 \\
9.8 \\
13.0 \\
9.0 \\
13.1\end{array}$ & \begin{tabular}{|l|}
0.99 \\
0.97 \\
1.22 \\
1.64 \\
0.94 \\
0.88 \\
0.91
\end{tabular} & $\begin{array}{l}0.56 \\
0.58 \\
0.87 \\
0.80 \\
0.90 \\
0.90 \\
0.62\end{array}$ & $\begin{array}{l}0.10 \\
0.12 \\
0.22 \\
0.14 \\
0.13 \\
0.12 \\
-\end{array}$ \\
\hline $\begin{array}{l}\text { Case } 3 \\
11 \text { days } \\
\text { birth wt. } \\
2,100 \text { grams }\end{array}$ & $\begin{array}{l}1 \\
2 \\
3 \\
4 \\
5\end{array}$ & $\begin{aligned} & \mathbf{0} \\
& \mathbf{0} \\
& \text { ACTH } \\
& \mathbf{8} \\
& \mathbf{0} \\
& \mathbf{0}\end{aligned}$ & $\begin{array}{l}2,200 \\
2,500 ? \\
2,450\end{array}$ & $\begin{array}{l}350 \\
345 \\
350 \\
355 \\
375\end{array}$ & $\begin{array}{l}13.5 \\
13.8 \\
14.0 \\
14.2 \\
15.0\end{array}$ & $\begin{array}{l}13.0 \\
12.8 \\
13.0 \\
13.2 \\
13.9\end{array}$ & $\begin{array}{l}185 \\
170 \\
142+ \\
155 \\
165\end{array}$ & $\begin{array}{l}6.1 \\
5.4 \\
5.7 \\
5.4 \\
6.9\end{array}$ & $\begin{array}{l}4.1 \\
3.9 \\
4.8 \\
3.4 \\
5.1\end{array}$ & & $\begin{array}{l}0.76 \\
0.60 \\
1.00 \\
0.88 \\
0.92\end{array}$ & $\begin{array}{l}0.21 \\
0.18 \\
0.25 \\
0.17 \\
0.19\end{array}$ \\
\hline $\begin{array}{l}\text { Case } 4 \\
19 \text { days } \\
\text { birth wt. } \\
1,800 \text { grams }\end{array}$ & $\begin{array}{l}1 \\
2 \\
3 \\
4 \\
5 \\
6\end{array}$ & $\begin{aligned} & \mathbf{0} \\
& \mathbf{0} \\
& \text { ACTH } \\
& 20 \\
& \mathbf{0} \\
& \mathbf{0} \\
& \mathbf{0}\end{aligned}$ & $\begin{array}{l}2,320 \\
2,400 \\
2,455\end{array}$ & $\begin{array}{l}390 \\
415 \\
420 \\
420 \\
420 \\
445\end{array}$ & $\begin{array}{l}15.6 \\
16.6 \\
16.8 \\
16.8 \\
16.8 \\
17.8\end{array}$ & $\begin{array}{l}14.4 \\
15.4 \\
15.6 \\
15.6 \\
15.6 \\
16.5\end{array}$ & $\begin{array}{l}150 \\
115 \\
150 \\
195 \\
130 \\
175\end{array}$ & $\begin{array}{r}9.5 \\
6.9 \\
6.4 \\
17.0 \\
10.0 \\
7.7\end{array}$ & $\begin{array}{r}9.7 \\
6.7 \\
12.3 \\
7.8 \\
7.5 \\
12.1\end{array}$ & $\begin{array}{l}0.55 \\
0.49 \\
1.03 \\
0.97 \\
0.72 \\
1.02\end{array}$ & $\begin{array}{l}0000 \\
0000 \\
1.20 \\
0.72 \\
0000 \\
0000\end{array}$ & $\begin{array}{l}\overline{0.18} \\
0.23 \\
\overline{-} \\
0.14\end{array}$ \\
\hline
\end{tabular}


ADRENOCORTICAL CONTROL OF NA \& K EXCRETION IN NEWBORNS

TABLE I-Continued

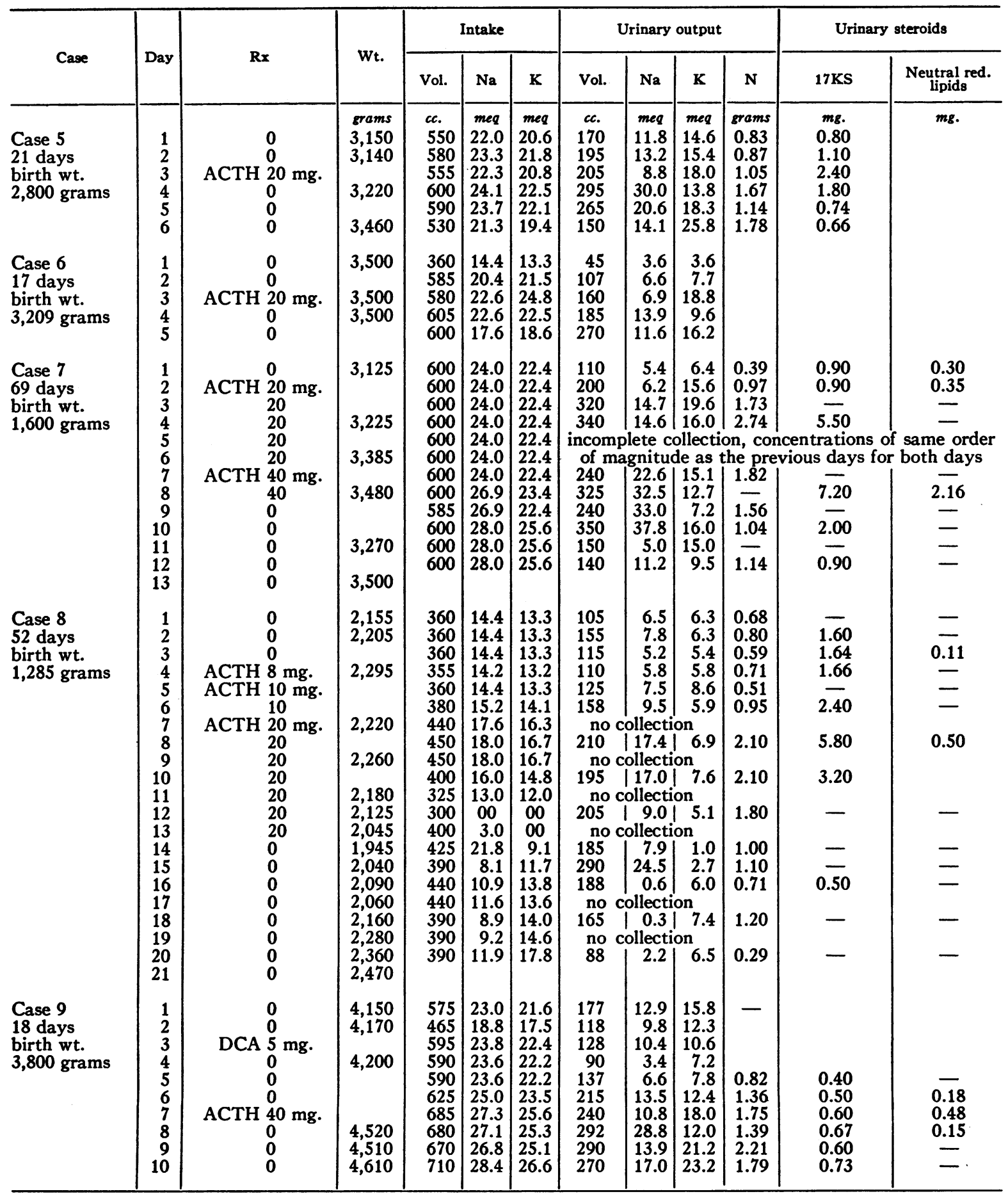


TABLE I-Continued

\begin{tabular}{|c|c|c|c|c|c|c|c|c|c|c|c|c|}
\hline \multirow{2}{*}{ Case } & \multirow{2}{*}{ Day } & \multirow{2}{*}{$\mathbf{R x}$} & \multirow{2}{*}{ Wt. } & \multicolumn{3}{|c|}{ Intake } & \multicolumn{4}{|c|}{ Urinary output } & \multicolumn{2}{|c|}{ Urinary steroids } \\
\hline & & & & Vol. & $\mathrm{Na}$ & $\mathbf{K}$ & Vol. & $\mathrm{Na}$ & $\mathbf{K}$ & $\mathbf{N}$ & $17 \mathrm{KS}$ & $\underset{\text { lipids }}{\text { Neutral red. }}$ \\
\hline $\begin{array}{l}\text { Case } 10 \\
21 \text { days } \\
\text { birth wt. } \\
1,210 \text { grams }\end{array}$ & $\begin{array}{r}1 \\
2 \\
3 \\
4 \\
5 \\
6 \\
7 \\
8 \\
9 \\
10 \\
11\end{array}$ & $\begin{aligned} & 0 \\
& 0 \\
& \text { DCA } 5 \mathrm{mg} . \\
& 0 \\
& 0 \\
& 0 \\
& 0 \\
& \text { ACTH } 20 \mathrm{mg} . \\
& 0 \\
& 0 \\
& 0\end{aligned}$ & $\begin{array}{l}\text { grams } \\
1,560 \\
1,645 \\
\\
1,760 \\
1,850 \\
1,860 \\
2,050\end{array}$ & \begin{tabular}{l|}
$c c$. \\
210 \\
210 \\
220 \\
226 \\
238 \\
240 \\
240 \\
260 \\
270 \\
295
\end{tabular} & \begin{tabular}{|c|} 
meq \\
10.9 \\
10.9 \\
11.4 \\
11.8 \\
12.4 \\
12.5 \\
12.5 \\
13.5 \\
14.1 \\
15.4
\end{tabular} & \begin{tabular}{|r|} 
meq \\
8.2 \\
8.2 \\
8.6 \\
8.8 \\
9.3 \\
9.4 \\
9.4 \\
10.1 \\
10.8 \\
11.8
\end{tabular} & $\begin{array}{l}c c . \\
60+ \\
96 \\
53 \\
50 \\
50 \\
65 \\
90 \\
60 \\
150+ \\
100\end{array}$ & $\begin{array}{r}m e q \\
5.4 \\
7.2 \\
3.4 \\
2.8 \\
3.3 \\
4.8 \\
9.0 \\
4.4 \\
18.3 \\
4.7\end{array}$ & \begin{tabular}{c|}
$m e q$ \\
3.3 \\
4.3 \\
2.9 \\
3.2 \\
2.9 \\
2.6 \\
4.4 \\
6.1 \\
2.7 \\
7.0
\end{tabular} & grams & $\begin{array}{l}0.33 \\
0.20 \\
0.73 \\
0.60 \\
-\end{array}$ & $m g$. \\
\hline $\begin{array}{l}\text { Case } 11 \\
20 \text { days } \\
\text { birth wt. } \\
1,250 \text { grams }\end{array}$ & $\begin{array}{r}1 \\
2 \\
3 \\
4 \\
5 \\
6 \\
7 \\
8 \\
9 \\
10\end{array}$ & $\begin{aligned} & 0 \\
& 0 \\
& \text { ACTH } 20 \mathrm{mg} . \\
& 0 \\
& 0 \\
& 0 \\
& \text { DCA } 2 \mathrm{mg} . \\
& 0 \\
& 0 \\
& 0\end{aligned}$ & $\begin{array}{l}1,460 \\
1,560 \\
1,565 \\
1,655 \\
1,715\end{array}$ & $\begin{array}{l}210 \\
218 \\
230 \\
240 \\
250 \\
267 \\
270 \\
270 \\
270 \\
270\end{array}$ & $\begin{array}{l}13.8 \\
14.2 \\
15.2 \\
15.8 \\
16.4 \\
17.6 \\
17.8 \\
17.8 \\
17.8 \\
17.8\end{array}$ & $\begin{array}{l}11.5 \\
12.0 \\
12.6 \\
13.2 \\
13.7 \\
14.7 \\
14.8 \\
14.8 \\
14.8 \\
14.8\end{array}$ & $\begin{array}{r}100 \\
110 \\
115 \\
160 \\
98 \\
160 \\
125 \\
73 \\
68 \\
78\end{array}$ & $\begin{array}{r}7.0 \\
8.2 \\
7.4 \\
17.6 \\
8.1 \\
4.7 \\
8.0 \\
5.9 \\
7.3 \\
8.4\end{array}$ & $\begin{array}{l}6.0 \\
8.5 \\
8.0 \\
7.0 \\
5.7 \\
8.4 \\
9.0 \\
9.0 \\
5.2 \\
5.8\end{array}$ & $\begin{array}{l}0.62 \\
0.66 \\
0.73 \\
1.12 \\
0.62 \\
0.50 \\
0.75\end{array}$ & $\begin{array}{l}0.70 \\
0.60 \\
0.60 \\
1.20 \\
0.40 \\
0.40\end{array}$ & $\begin{array}{l}\overline{0.15} \\
0.26 \\
0.41 \\
\overline{0.09}\end{array}$ \\
\hline $\begin{array}{l}\text { Case } 12 \\
18 \text { days } \\
\text { birth wt. } \\
1,630 \text { grams }\end{array}$ & $\begin{array}{l}1 \\
2 \\
3 \\
4 \\
5 \\
6 \\
7\end{array}$ & $\begin{array}{cl}0 \\
0 \\
0 \\
0 \\
\text { DCA } \begin{array}{c}1 \mathrm{mg} . \\
0 \\
0\end{array}\end{array}$ & $\begin{array}{l}2,130 \\
2,195 \\
2,325\end{array}$ & $\begin{array}{l}270 \\
320 \\
330 \\
320 \\
325 \\
330\end{array}$ & $\begin{array}{l}10.8 \\
12.8 \\
13.2 \\
12.8 \\
13.0 \\
13.2\end{array}$ & $\begin{array}{l}10.0 \\
11.9 \\
12.2 \\
11.9 \\
12.0 \\
12.2\end{array}$ & $\begin{array}{l}138 \\
145 \\
165 \\
100 \\
164 \\
150\end{array}$ & $\begin{array}{r}8.0 \\
8.2 \\
9.0 \\
7.2 \\
11.0 \\
8.4\end{array}$ & $\begin{array}{l}6.3 \\
9.4 \\
9.4 \\
6.8 \\
9.0 \\
8.7\end{array}$ & & & \\
\hline $\begin{array}{l}\text { Case } 13 \\
22 \text { days } \\
\text { birth wt. } \\
1,540 \text { grams }\end{array}$ & $\begin{array}{l}1 \\
2 \\
3 \\
4 \\
5 \\
6 \\
7 \\
8 \\
9\end{array}$ & $\begin{array}{c}0 \\
0 \\
0 \\
\text { Cortisone } 50 \mathrm{mg} . \\
0 \\
0 \\
0 \\
0 \\
0\end{array}$ & $\begin{array}{l}1,860 \\
1,940 \\
2,005 \\
2,075\end{array}$ & $\begin{array}{l}265 \\
280 \\
295 \\
290 \\
300 \\
300 \\
300 \\
295\end{array}$ & $\begin{array}{l}13.9 \\
14.7 \\
15.5 \\
15.3 \\
15.8 \\
15.8 \\
15.8 \\
15.5\end{array}$ & $\begin{array}{l}10.5 \\
11.0 \\
11.6 \\
11.4 \\
11.9 \\
11.9 \\
11.9 \\
11.6\end{array}$ & $\begin{array}{r}84 \\
85 \\
106 \\
93 \\
130 \\
155 \\
84 \\
118\end{array}$ & $\begin{array}{l}6.0 \\
5.5 \\
7.6 \\
7.0 \\
7.8 \\
9.9 \\
5.7 \\
7.3\end{array}$ & $\begin{array}{l}5.6 \\
5.3 \\
6.3 \\
6.3 \\
7.3 \\
7.4 \\
5.1 \\
6.7\end{array}$ & $\begin{array}{l}0.27 \\
0.35 \\
0.34 \\
0.36 \\
0.50 \\
0.49 \\
0.38 \\
0.46\end{array}$ & $\begin{array}{l}0.85 \\
1.06 \\
1.14 \\
1.22 \\
0.93 \\
0.16 \\
0.06\end{array}$ & $\begin{array}{l}\overline{0.14} \\
0.13 \\
0.18 \\
\overline{0.09} \\
-\end{array}$ \\
\hline $\begin{array}{l}\text { Case } 14 \\
25 \text { days } \\
\text { birth wt. } \\
1,455 \text { grams }\end{array}$ & $\begin{array}{r}1 \\
2 \\
3 \\
4 \\
5 \\
6 \\
7 \\
8 \\
9 \\
10 \\
11 \\
12 \\
13 \\
14\end{array}$ & $\begin{array}{c}0 \\
0 \\
\text { Cortisone } 25 \mathrm{mg} . \\
0 \\
0 \\
0 \\
0 \\
\text { ACTH } 30 \mathrm{mg} . \\
0 \\
0 \\
0 \\
0 \\
0 \\
0\end{array}$ & $\begin{array}{l}1,650 \\
1,735 \\
1,825 \\
1,950 \\
1,965 \\
\\
2,025 \\
2,105\end{array}$ & $\begin{array}{l}240 \\
240 \\
240 \\
240 \\
265 \\
270 \\
270 \\
280 \\
300 \\
325 \\
330 \\
330 \\
330\end{array}$ & $\begin{array}{l}16.3 \\
16.3 \\
16.3 \\
16.3 \\
18.0 \\
18.3 \\
18.3 \\
19.0 \\
20.4 \\
22.0 \\
22.5 \\
22.5 \\
22.5\end{array}$ & $\begin{array}{l}13.4 \\
13.4 \\
13.4 \\
13.4 \\
14.8 \\
15.1 \\
15.1 \\
15.7 \\
16.8 \\
18.2 \\
18.5 \\
18.5 \\
18.5\end{array}$ & $\begin{array}{r}116 \\
110 \\
40 \\
70 \\
73 \\
111 \\
85 \\
117 \\
120+ \\
120 \\
95 \\
117 \\
133\end{array}$ & $\begin{array}{r}8.9 \\
7.5 \\
2.9 \\
2.9 \\
3.5 \\
7.5 \\
6.0 \\
6.1 \\
12.5 \\
12.4 \\
4.3 \\
9.1 \\
11.0\end{array}$ & $\begin{array}{r}9.6 \\
8.3 \\
3.0 \\
2.7 \\
4.5 \\
7.2 \\
6.1 \\
9.7 \\
6.6 \\
7.0 \\
10.5 \\
10.3 \\
10.9\end{array}$ & $\begin{array}{l}0.54 \\
0.46 \\
0.22 \\
0.25 \\
0.26 \\
0.44 \\
0.38 \\
0.57 \\
0.75 \\
0.53 \\
0.89 \\
0.78 \\
0.80\end{array}$ & $\left\{\begin{array}{c}0.77 \\
0.70 \\
3 \text { day aver. } \\
0.71 \\
0.84 \\
0.58 \\
0.73 \\
0.74 \\
0.64 \\
0.51 \\
0.63 \\
0.77\end{array}\right.$ & 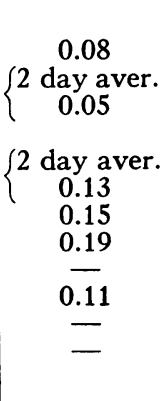 \\
\hline $\begin{array}{l}\text { Case } 15 \\
6 \text { months } \\
\text { birth wt. } \\
3,500 \text { grams }\end{array}$ & $\begin{array}{l}1 \\
2 \\
3 \\
4 \\
5 \\
6\end{array}$ & $\begin{aligned} & 0 \\
& 0 \\
& \text { ACTH } 20 \mathrm{mg} . \\
& 0 \\
& 0 \\
& 0\end{aligned}$ & $\begin{array}{l}7,020 \\
7,100\end{array}$ & $\begin{array}{r}1,025 \\
1,025 \\
1,025 \\
960 \\
730 \\
1,000\end{array}$ & $\begin{array}{l}52.0 \\
52.0 \\
50.0 \\
50.0 \\
34.0 \\
52.0\end{array}$ & $\begin{array}{l}55.0 \\
55.0 \\
54.0 \\
52.0 \\
36.0 \\
55.0\end{array}$ & $\begin{array}{l}370 \\
415 \\
260 \\
535 \\
235 \\
225\end{array}$ & $\begin{array}{l}32.0 \\
37.0 \\
13.0 \\
59.0 \\
14.0 \\
33.0\end{array}$ & $\begin{array}{l}36.0 \\
41.0 \\
39.0 \\
30.0 \\
33.0 \\
31.0\end{array}$ & & $\begin{array}{l}0.70 \\
0.50 \\
0.70 \\
0.90 \\
0.50 \\
0.50\end{array}$ & \\
\hline
\end{tabular}


ACTH was discontinued. Sodium concentration in the urine never exceeded $87 \mathrm{meq} / \mathrm{l}$.

In comparison with the infants of the younger age group a study was made on one six month old full term infant (Case 15). He showed sodium retention when given $\mathrm{ACTH}$ for one day in a manner entirely similar to that shown by the usual adult receiving $\mathrm{ACTH}$ for one day.

Figures for the intake and excretion of ions and for the excretion of steroids and nitrogen in these cases are given in Table I. Figure 1 compares the electrolyte balance of a characteristic newborn given ACTH and also DCA with that of a six month old infant given ACTH.

\section{DISCUSSION}

The functional immaturity of the kidney of the infant is well known having been elucidated by McCance and Young (8), Rubin, Bruck and Rapoport (9), and others. However, it is difficult to account for this peculiar electrolyte response to ACTH on this basis since the administration of DCA to the newborn caused decreased excretion of sodium as it does in the older child and adult. This suggests that the kidney of the newborn is capable of responding normally to adrenocortical hormone. It seems more probable that the peculiarity of electrolyte excretion is due to a difference in the hormones secreted by the immature adrenal. This might be due either to the secretion of a hormone not present in later life or to an alteration in the balance between hormones having varying quantitative or qualitative effects on sodium excretion. The latter alternative might be considered on the basis of the theory advanced by Thorn and associates (10) suggesting that weak sodium retainers such as Compound $E$ and $F$ compete with potent sodium retainers such as DCA and thereby induce salt loss. Thus it is possible that in the case of the newborn the adrenal when stimulated by ACTH may secrete relatively more Compound F-like hormone than the adult adrenal under similar circumstances. In patients with congenital hyperplasia the ACTH-induced secretion of androgenic steroids might also compete with DCA-like steroids, and thereby induce the observed sodium loss $(6,11)$.

In our small series there was no demonstrable difference in response between premature and full term infants of the same post-natal age, but it is possible that the abnormal effects of the adrenal on electrolyte excretion persist longer in premature than in full term infants.

\section{SUMMARY AND CONCLUSIONS}

Twelve newborn infants were given ACTH for periods of one day to ten days. This was regularly accompanied by the expected increase in the excretion of neutral reducing lipid, 17-ketosteroids, and urinary nitrogen. The urinary excretion of potassium showed only moderate changes, usually increasing on the day of ACTH injection as expected from adult experience. Sodium excretion was always increased one day after an adequate dose of ACTH was given. This frequently was of sufficient magnitude to exceed the dietary intake of this ion. One six month old infant retained sodium after the administration of $\mathrm{ACTH}$ in the same manner as adults. When DCA was administered to newborns they reacted like adults, showing a decreased excretion of sodium. In one newborn, $25 \mathrm{mg}$. of cortisone produced sodium retention; $50 \mathrm{mg}$. of cortisone given to another newborn produced no effect on the sodium balance.

\section{ACKNOWLEDGMENTS}

The technical assistance of Mr. Henry Schulte and Mrs. Mary Ellen Crafton in carrying out the steroid and nitrogen determinations is gratefully acknowledged.

The author wishes to thank Dr. Lawson Wilkins for his advice and encouragement in the carrying out of the study and for his help in the preparation of the manuscript.

\section{REFERENCES}

1. Jaudon, J. C., Hypofunction of adrenals in early life. J. Pediat., 1946, 29, 696.

2. Venning, E., The effect of ACTH during neonatal period, in Proceedings of the First Clinical ACTH Conference, J. R. Mote, Editor. The Blakiston Co., Philadelphia, 1950, p. 25.

3. Klein, R., and Hanson, J., Adrenocortical function in the newborn as measured by adrenocorticotropic hormone-eosinophil response. Pediatrics, in press.

4. Klein, R., Discussion, in Venning, E., The effect of ACTH during the neonatal period, in Proceedings of the First Clinical ACTH Conference, J. R. Mote, Editor. The Blakiston Co., Philadelphia, 1950, p. 30 . 
5. Randolph, T. G., Differentiation of leukocytes in counting chamber by propylene glycol-aqueous stains; screen for detection of major blood abnormality. Am. J. Clin. Path., Tech. Sect., 1944, 8, 48.

6. Lewis, R. A., and Wilkins, L., The effect of adrenocorticotrophic hormone in congenital adrenal hyperplasia with virilism and in Cushing's syndrome treated with methyl testosterone. J. Clin. Invest., 1949, 28, 394.

7. Wallace, W., Personal communication.

8. McCance, R. A., and Young, W. F., The secretion of urine by newborn infants. J. Physiol., 1941, 99, 265.
9. Rubin, M. I., Bruck, E., and Rapoport, M., Maturation of renal function in childhood; clearance studies. J. Clin. Invest., 1949, 28, 1144.

10. Thorn, G. W., Forsham, P. H., Bennett, L. L., Roche, M., Reiss, R. S., Slessor, A., Flink, E. B., and Somerville, W., Clinical and metabolic changes in Addison's disease following the administration of compound E acetate. Tr. A. Am. Physicians, 1949, 62, 233.

11. Wilkins, L., Klein, R., and Lewis, R. A., The response to ACTH in various types of adrenal hyperplasia, in Proceedings of the First Clinical ACTH Conference, J. R. Mote, Editor. The Blakiston Co., Philadelphia, 1950, p. 184. 